\title{
Avoiding chaos in granular dampers
}

\author{
María Victoria Ferreyra ${ }^{1, *}$, Julián M. Gómez-Paccapelo ${ }^{1}$, Ramiro Suarez ${ }^{1}$, and Luis A. Pugnaloni ${ }^{1,2}$ \\ ${ }^{1}$ Dpto. de Física, Facultad de Ciencias Exactas y Naturales, Universidad Nacional de La Pampa, Uruguay 151, 6300 Santa Rosa (La \\ Pampa), Argentina. \\ ${ }^{2}$ Consejo Nacional de Investigaciones Científicas y Técnicas, Argentina.
}

\begin{abstract}
Granular dampers are passive devices used to attenuate mechanical vibrations. The most common configuration consists in an enclosure, partially filled with particles, attached to the vibrating structure that needs to be damped. The energy is dissipated due to inelastic collisions and friction between the grains and between the grains and the inner walls of the container as the structure vibrates. As a result of the collisions, the mechanical response of the system often results in chaotic motion even if the driving is harmonic. Despite the vibration attenuation achieved, this chaotic response may render the granular damper unsuitable for a range of applications. In this work, we showcase two simple modifications of the enclosure design that are able to mitigate the chaotic response of the granular damper. To this end we use Discrete Element Method simulations of: (a) a granular damper with a conical base, and (b) a granular damper with obstacles fixed inside the enclosure. We compare results against a standard flat-base enclosure damper. The basic mechanical response of the dampers is characterized by measuring the apparent mass and the loss factor. The suppression of the chaotic response is assessed qualitatively via the phase space diagram.
\end{abstract}

\section{Introduction}

The attenuation of vibrations is of vital importance in most mechanical systems: vehicles, aircrafts, mechanical tools, aerospace structures and civil structures. The most common way of damping vibrations is through viscous fluids or viscoelastic materials. The conventional viscous (Newtonian) damper has a well known behavior: the damping force is proportional to the relative velocity of two moving parts. Therefore, its behavior is predictable by solving a simple differential equation [1].

Granular dampers (GD), also known as particle dampers, constitute an alternative way of attenuating vibrations. Such devices are enclosures partially filled with granular material which are attached to the primary vibrating system [2]. During vibration, the primary system transfers momentum to the particles. Inside the GD, the energy is dissipated due to inelastic collisions and friction between the grains and between the grains and the inner walls of the container, where the kinetic energy is converted into heat. The simplicity, affordability and low maintenance of GDs are features that make them attractive (see [3] for a recent review). Since the main mechanisms of dissipation are insensitive to temperature variations, GDs are very effective in extreme environments where the usual viscous dampers lose their operational capacity. Even in absence of gravity, GDs are able to attenuate vibrations [4-6].

Unfortunately, the multiple collisions between particles and between particles and walls, apart from energy

\footnotetext{
*e-mail: ferreyravic@gmail.com
}

dissipation, lead to a nonlinear mechanical response [7]. This poses a major challenge to the design of a GD since small changes in the operating conditions may lead to significant deviations from the expected response. Previous authors have tried to tackle this issue by introducing an additional component (a foam) inside the GD [8]. More challenging yet is the fact that the dynamics of a GD develops chaotic regimes for some ranges of the control parameters [7].

This work is focused on suppressing the chaotic behavior of the mechanical response of GDs. By using simulations based on the Discrete Element Method (DEM) $[9,10]$, we propose different strategies to reduce the nonlinearity of the dynamics. We compare a standard GD (a prismatic enclosure) with: (a) a GD with a conical base, and (b) a GD with fixed scattered obstacles inside. Although previous studies have tested different enclosure shapes [11], these were not aimed at assessing the suppression of chaotic behaviour. Our simulations show that the new modified enclosures can indeed prevent chaotic dynamics while conserving a good level of vibration attenuation.

\section{Model and simulations}

Our system consists of a single degree of freedom (SDoF) model constrained to move in the vertical direction where a granular damper is carved in the primary mass, as it is sketched in Fig. 1. A full description of the model and simulation can be found in [12]. The SDoF model has a primary system of mass $M=2.37 \mathrm{~kg}$, attached to an 

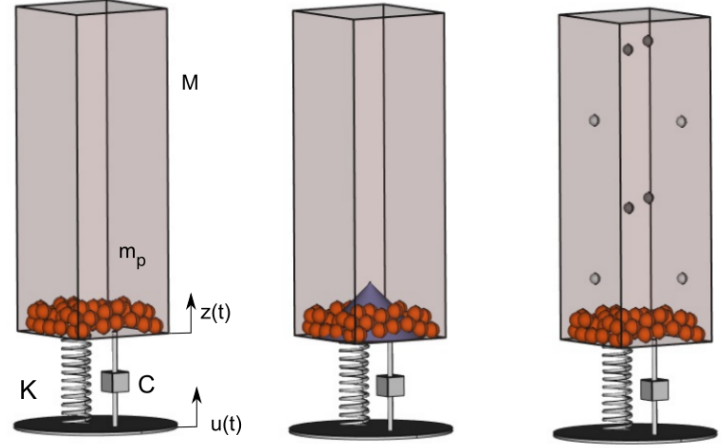

Figure 1. Model of the SDoF system with a granular damper. (Left) Flat enclosure. (Center) Enclosure with conical floor. (Right) Enclosure with obstacles.

oscillating platform by a spring of stiffness $K=21500$ $\mathrm{Nm}^{-1}$ and a viscous damper with a small damping constant $C=7.6 \mathrm{Nsm}^{-1}$. The primary mass body is hollow and is partially filled with spherical particles that are free to move in all directions inside this enclosure. For $C=0$ and without particles, the natural frequency of the system is $f_{0}=\sqrt{K / M} / 2 \pi \simeq 15.16 \mathrm{~Hz}$.

The system is excited by imposing a harmonic vertical motion to the platform, whose position is described as $u(t)=U \cos \omega t$, where $U$ is the amplitude and $\omega$ is the angular frequency. Both $U$ and $\omega$ are our control parameters. The acceleration of gravity is set to $g=9.8 \mathrm{~ms}^{-2}$ in the downward direction.

The basic cavity with particles is a prismatic enclosure with square base of $12.25 r \times 12.25 r$, where $r$ is the radius of one particle. The height was set to $36.5 r$. We have simulated three different types of GDs (see Fig. 1): (1) with flat floor and ceiling, (2) with a conical floor of height $12.25 r$ and diameter $12.25 r$, and (3) with flat floor and ceiling but with 8 fixed obstacles inside. The obstacles are spheres of diameter $0.5 r$ placed at 4 layers equally spaced. Each layer has 2 obstacles near opposite corners and the corners used are alternated from layer to layer. In the case of the conical base we have raised the position of the ceiling so that the total volume of the enclosure matches that of the flat floor GD.

The motion of the primary system is described by the differential equation

$$
M \ddot{z}(t)+C \dot{z}(t)+K z(t)=C \dot{u}(t)+k u(t)+F_{\text {part }}(t),
$$

where $z(t)$ is the position of the primary mass, $u(t)$ is the position of the oscillating platform and $F_{\text {part }}$ is the total force exerted by the particles against the inner walls of the container in the vertical direction. Equation (1) can be solved numerically once $F_{\text {part }}$ is obtained via DEM simulations as described below $[9,10]$.

The GDs are partially filled with 250 spherical particles with total mass $m_{p}=0.227 \mathrm{~kg}$. The volume fraction in the enclosure is 0.191 . The interaction between two particles, between a particle and an obstacle and between a particle and a wall is modeled by normal and tangential components of the contact force applied

\begin{tabular}{cc}
\hline \hline Property & Value \\
\hline Young's modulus $E$ & $2.03 \times 10^{11} \mathrm{Nm}^{-2}$ \\
Density & $8030 \mathrm{kgm}^{-3}$ \\
Poisson's ratio $v$ & 0.28 \\
Friction coefficient $\mu_{\mathrm{d}}$ & 0.3 \\
Normal damping coefficient $\gamma_{\mathrm{n}}$ & $3.660 \times 10^{3} \mathrm{kgs}^{-1} \mathrm{~m}^{-1 / 2}$ \\
Shear damping coefficient $\gamma_{\mathrm{s}}$ & $1.098 \times 10^{4} \mathrm{~kg} \mathrm{~s}^{-1} \mathrm{~m}^{-1 / 2}$ \\
Time step $\delta t$ & $8.75 \times 10^{-8} \mathrm{~s}$ \\
Time of simulation & $>8.75 \mathrm{~s}$ \\
Particle radius $R$ & $0.003 \mathrm{~m}$ \\
Total particle mass $m_{\mathrm{p}}$ & $0.227 \mathrm{~kg}$ \\
Volume fraction & 0.191 \\
\hline
\end{tabular}

Table 1. Simulation parameters and material properties of the particles.

at the contact point. The normal component corresponds to the Hertz-Kuwabara-Kono model $\left(F_{\mathrm{n}}=-k_{\mathrm{n}} \alpha^{3 / 2}-\right.$ $\gamma_{\mathrm{n}} v_{\mathrm{n}} \sqrt{\alpha}$ ), where $\alpha$ is the particle-particle overlap and $v_{\mathrm{n}}$ the normal component of the relative velocity at the contact [10]. For the tangential component we use $F_{\mathrm{t}}=$ $-\min \left(\left|\gamma_{\mathrm{t}} v_{\mathrm{t}} \sqrt{\alpha}\right|,\left|\mu_{\mathrm{d}} F_{\mathrm{n}}\right|\right) \operatorname{sgn}\left(v_{\mathrm{t}}\right)$, where $v_{\mathrm{t}}$ is the tangential component of the relative velocity at the contact [13]. The material parameter $k_{\mathrm{n}}$ can be obtained from the Young's modulus, Poisson's ratio and the radius of the interacting bodies [10]. We use values compatible with steel as listed in Table 1.

Equation (1), and the degrees of freedom of all the particles interacting via the forced described above, are integrated using the velocity Verlet algorithm [14]. Rotations are managed using quaternions [14]. For the relatively large particles we consider here, the effect of the surrounding air can be neglected. Therefore, we do not simulate this aerodynamic effect.

\section{Loss factor and apparent mass}

To analyze the mechanical response of the GD we use the loss factor and the apparent mass of the grains, following the definitions proposed by Salueña et al. [15] and Masmoudi et al. [16]. The loss factor $\eta$ is the ratio between the energy $E_{d}$ dissipated per cycle and the maximum kinetic energy $E_{k}$ achieved during a cycle. This can be written in terms of the phase shift between the fundamental components of the displacement $z(t)$ of the GD and the external force $F(t)$ exerted on it,

$$
\eta(\omega)=\tan \phi(\omega) .
$$

Here, $\phi=\phi_{z}-\phi_{F}$ is the loss angle at driving frequency $\omega$. In practice, we measure $\phi_{z}$ from the phase of the acceleration $\Gamma(t), \phi_{z}=\phi_{\Gamma}+\pi$.

We obtain the phases $\phi_{\Gamma}$ and $\phi_{F}$ and the magnitudes $A_{F}$ and $A_{\Gamma}$ of the fundamental component of the Fourier series of the acceleration and force signals as

$$
\begin{aligned}
A_{F} e^{j \phi_{F}} & =\frac{1}{n T} \int_{0}^{n T} F(t) e^{-j \omega t} d t, \\
A_{\Gamma} e^{j \phi_{\Gamma}} & =\frac{1}{n T} \int_{0}^{n T} \Gamma(t) e^{-j \omega t} d t,
\end{aligned}
$$




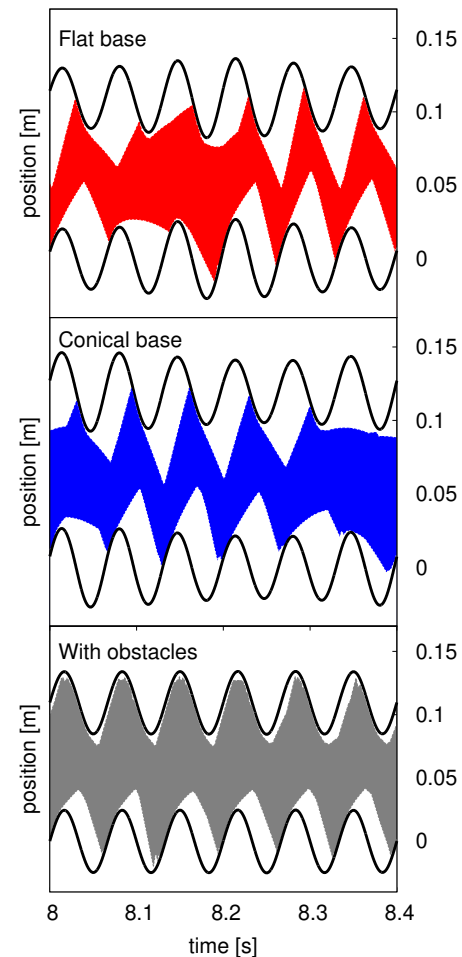

Figure 2. Trajectory of the enclosure and the granular bed for $U=0.75 r$ and $15 \mathrm{~Hz}$ for the three GDs considered (see labels). The upper and lower solid line indicate the position of the floor and ceiling of the enclosure. The shaded area corresponds to the vertical spread of the grains inside represented by the highest and lowest particle at each time.

where $n$ is the number of cycles and $T=2 \pi / \omega$ is the period of the driving of the vibrating platform.

The apparent mass of the system of grains is calculated from the amplitudes of $F(t)$ and $\Gamma(t)$,

$$
m=A_{F} / A_{\Gamma}-M,
$$

where $M$ is the primary mass (the mass of the enclosure itself).

\section{Results}

Figure 2 shows the behavior of the granular system inside the enclosure at $U=0.75 r$ and $f=15 \mathrm{~Hz}$ (close to resonance), for the three different GDs studied. As we can see, for this particular driving parameters, the motion of the grains looks periodic for the GD with obstacles but chaotic for the other GDs. This type of dynamics has been described before for the flat GD [7]. However, we will focus on the dynamics of the enclosure itself rather than the particles, since the ultimate goal of a GD is to achieve a particular response of the primary system.

The basic mechanical response of the different GDs is shown in Fig. 3 where we plot the apparent mass $m$ and the loss factor $\eta$ as a function of the scaled acceleration $A_{\Gamma} / g$ of the primary mass. Since the driving frequency is close

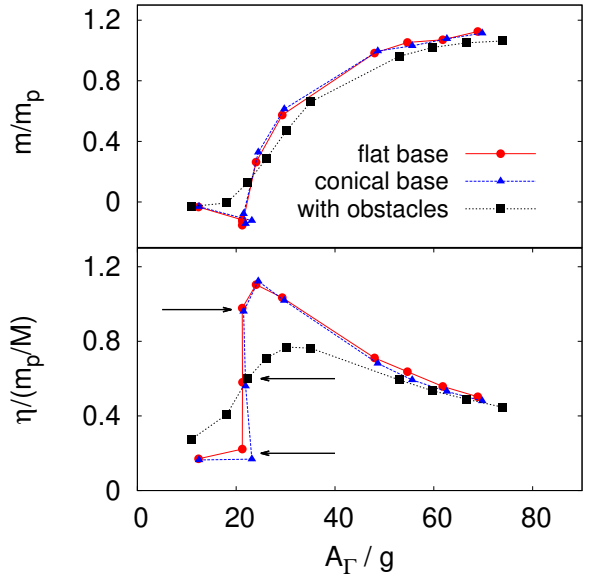

Figure 3. Apparent mass $m / m_{\mathrm{p}}$ of the grains (top) and loss factor $\eta$ (bottom) as a function of the amplitude of the acceleration $A_{\gamma}$, for $f=15 \mathrm{~Hz}$. Different $A_{\Gamma}$ are obtained by varying $U$. Results for different GDs (see legends).

to resonance, the accelerations achieved are high. The GD with obstacles show a smoother curve, especially for the loss factor. However, the peak value for $\eta$ is $50 \%$ lower than for the other two GDs.

Let us focus our attention on the results for $U=0.5 r$, $0.75 r$ and $1.0 r\left(18<A_{\Gamma} / g<26\right)$, which are indicated by arrows in Fig. 3. For the flat and conical base GDs the apparent mass of the grains is negative at these drivings. The occurrence of negative masses has been discussed in detail by Sánchez et al. [12] and Ferreyra et al. [17] as a result of the synchronization of the collisions with a particular phase of the enclosure oscillation. The flat and conical base GDs often show that particles move collectively and reach the floor and the ceiling in a solid-like (rather compact) granular state. The GD with obstacles disperses the granular pack into a "gas" and the ceiling is reached by the grains in a softer way (see Fig. 2).

To assess if the motion of the primary system is periodic, quasi-periodic or chaotic, we plot in Fig. 4 the phase diagram (velocity of the primary system as a function of its position) for the three values of $U$ of interest. As we can see, the flat GD (red) shows a seemingly chaotic behaviour for $U=0.5 r$ and $U=0.75 r$ but becomes periodic at $U=1.0 r$. The conical base GD only displays chaos for $U=0.75 r$, whereas the GD with obstacles yields a periodic response in all the cases. This suggests that the two new designs proposed can in fact prevent chaos in some cases. It is worth mentioning that the three examples in Fig. 4 where a chaotic motion is apparent are the only cases found in our full set of simulations. However, Sánchez et al. have shown that there exist various regions of the parameter space where the flat GD can display chaos, at least in terms of the internal motion of the center of mass of the granular pack [7]. A more exhaustive exploration of $U$ and $f$ may show that the flat base GD enters into chaotic motion under several conditions. It 


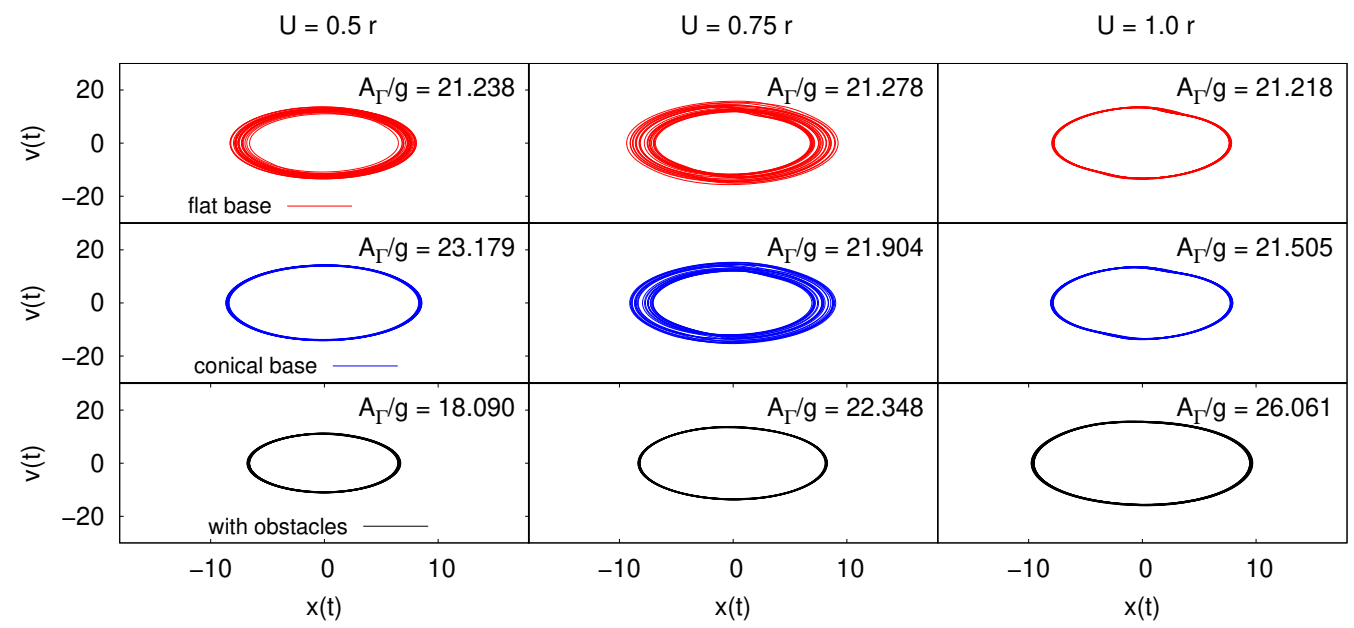

Figure 4. Phase diagram for the different GDs at $U=0.5,0.75$ and 1.0r.

would be interesting to see how the GD with obstacles, in particular, performs in such cases.

\section{Conclusions}

We have considered GDs that are modified with respect to the traditional simple enclosures by using a conical floor or by inserting obstacles in the cavity. We have shown that these simple changes in the design can alter significantly the dynamics and prevent the development of chaotic responses. In particular, the use of obstacles seems to avoid chaos at all amplitudes tested for a frequency close to resonance. Unfortunately, some of the damping efficiency is lost. However, dissipation is still considerable. Moreover, the apparent mass and loss factor become smoother functions of the driving amplitude, which is a desirable feature for the design engineer. It is important to emphasize that a rigorous proof of the existence or not of chaos requires a quantitative assessment using the Lyapunov's exponents. This will be pursued in the future together with an exhaustive research on the parameter space of the driving.

\section{Acknowledgements}

We thank Martín Sánchez, Manuel Carlevaro, Marcos Madrid and Stéphane Job for valuable discussions. This work was funded by ANPCyT (Argentina) through grant PICT-2016-2658 and CAFCI (Argentina-France).

\section{References}

[1] S. Braun, D. Ewins, S.S. Rao. Encyclopedia of vibration (Academic Press, London, 2002)

[2] H.V. Panossian. J. Vib. Acoustics 114, 101-105 (1992)
[3] Z. Lu, S.F. Masri, X. Lu. Origination, development and applications of particle damping technology. In: Particle damping technology based structural control (Springer, Singapore, 2020)

[4] M.N. Bannerman, J.E. Kollmer, A. Sack, M. Heckel, P. Mueller, T. Pöschel. Phys. Rev. E 84, 011301 (2011)

[5] A. Sack, M. Heckel, J.E. Kollmer, F. Zimber, T. Pöschel. Phys. Rev. Lett. 111, 018001 (2013).

[6] H. Torres Menéndez, A. Sack., T. Pöschel. Granul. Matter 22, 1 (2020)

[7] M. Sánchez, M. Carlevaro. J. Sound Vib. 332, 2070 (2013)

[8] C. Wong, J. Rongong, AIAA J. 47, 953 (2009)

[9] P.A. Cundall, O.D.L. Strack. Geotechnique 29, 47 (1979)

[10] T. Pöschel, T. Schwager, Computational granular dynamics: Models and algorithms (Springer-Verlag, Berlin-Heidelberg, 2005)

[11] C. Wong, A. Spencer, J. Rongong, Effects of enclosure geometry on particle damping performance, In 50th AIAA/ASME/ASCE/AHS/ASC Structures, Structural Dynamics, and Materials Conference. AIAA, Palm Spring, California, USA (2009)

[12] M. Sánchez, L.A. Pugnaloni, J. Sound Vib. 330, 5812 (2011)

[13] J. Schafer, S. Dippel, D.E. Wolf, J. Physique I France 6, 5 (1996)

[14] M.P. Allen, D.J. Tildesley, Computer Simulation of Liquids. (Oxford Science Publications, Oxford,1989)

[15] C. Salueña, T. P oschel, S.E. Espiov, Phys. Rev. E 59, 4422 (1999)

[16] M. Masmoudi, S. Job, M.S. Abbes, I. Tawfiq, M. Haddar. Granul. Matter 18, 71 (2016)

[17] M.V. Ferreyra, M. Baldini, L.A. Pugnaloni, S. Job, arXiv:2007.07961 (2020) 